\title{
Structural distortion as prerequisite for superconductivity in LiFeAs
}

\author{
Ekkehard Krüger and Horst P. Strunk \\ Institut für Materialwissenschaft, Materialphysik, \\ Universität Stuttgart, D-70569 Stuttgart, Germany
}

(Dated: November 11, 2018)

\begin{abstract}
The nonadiabatic Heisenberg model predicts a structural distortion in LiFeAs below a temperature higher than (or at least equal to) the superconducting transition temperature. Within this grouptheoretical model, the reduction of the symmetry caused by the distortion is a prerequisite for the superconducting state in this compound and can be realized by a mere displacement of the iron atoms from their positions in the space group $P 4 / \mathrm{nmm}$.
\end{abstract}

Keywords: superconductivity, nonadiabatic Heisenberg model, group theory

\section{INTRODUCTION}

LiFeAs becomes superconducting at the "respectable" transition temperature $T_{c}=18 K$ without any chemical doping [1]. In this way LiFeAs differs remarkably from other high- $T_{c}$ FeAs compounds such as LaFeAsO which becomes superconducting only on (slight) doping with fluorine (or other specific dopants [2]). As a pure substance, however, LaFeAsO undergoes an antiferromagnetic spin-ordering transition at $\sim 137 K[3-6]$.

The magnetic and superconducting properties of LaFeAsO have been analyzed recently on the basis of the group-theoretical nonadiabatic Heisenberg model (NHM) 7, 8]. We reported evidence that both the antiferromagnetic and the superconducting state are connected with a magnetic 7, 9] and a superconducting [8, 10] band, respectively, of special symmetry. As an important consequence, it is the reduction of the symmetry of LaFeAsO, caused in this case by the doping, which activates superconductivity in this compound.

In the present paper we apply the NHM to LiFeAs and show, first, that the magnetic order observed in LaFeAsO is not stable in LiFeAs and, second, that in LiFeAs just as in LaFeAsO a superconducting state can be stable only when the symmetry of the crystal is reduced. In the case of LiFeAs, this reduction can be realized by a (small) displacement of the Fe atoms.

\section{COMPARISON OF THE BAND STRUCTURES OF LiFeAs AND LaFeAsO}

LiFeAs and LaFeAsO possess common properties facilitating the comparison of their band structures: both compounds possess the space group $P 4 / \mathrm{nmm}^{1}$, 3, 1115], the Fe atoms have the same positions in the unit cell, and the positions of the $\mathrm{Li}$ and As atoms in LiFeAs are group-theoretically equivalent to the positions of the La and As atoms in LaFeAsO. Solely the oxygen atoms are absent in LiFeAs. As a consequence, magnetic and superconducting bands in both compounds have the same symmetry as long as they are not related to the oxygen atoms.

Comparing the band structure of LiFeAs as depicted in Fig. 1 with the band structure of LaFeAsO (see Fig. 1 of Ref. [7] or [8]), we find both an important difference and analogies: On the one hand, LiFeAs does not possess a magnetic band related to the space group Imma as it exists in the band structure of LaFeAsO (Sec. II.1). On the other hand, both materials lack a superconducting band in the space group P4/nmm (Sec. II.2).

\section{II.1. The magnetic order observed in $\mathrm{LaFeAsO}$ is not stable in LiFeAs}

In stoichiometric LaFeAsO the magnetic band related to the space group Imma is evidently responsible for its observed [3 6] antiferromagnetic state [7]. In the band structure of LiFeAs, however, such a magnetic band does not exist. The reason is because the Bloch function with $Z_{1}$ symmetry disappears in the band structure of LiFeAs. This $Z_{1}$ state near the Fermi level, however, is an indispensable component of the magnetic band in LaFeAsO 7]. Hence, the symmetry of the Bloch functions of LiFeAs near the Fermi level is not compatible with the magnetic structure experimentally determined in LaFeAsO and, consequently, the magnetic order observed in LaFeAsO does not develop in LiFeAs. We cannot exclude, however, that other magnetic structures with other magnetic groups could be stable in distorted LiFeAs.

\section{II.2. Absence of a superconducting state in undistorted LiFeAs}

Undistorted LaFeAsO lacks a superconducting band because the two branches of any superconducting band in the space group $P 4 / \mathrm{nmm}$ are degenerated at point $M$ and are labeled by two representations $\Gamma^{+}$and $\Gamma^{-}$different with respect to the inversion 8]. Hence, the run of the bands on the lines $\Gamma X$ and $X M$ near the Fermi level of LaFeAsO and the symmetry of the Bloch functions at point $\Gamma$ do not allow the construction of a superconducting band in LaFeAsO [8].

In LiFeAs, the run of the bands on the lines $\Gamma X$ and $X M$ near the Fermi level is similar to the run of the 
related bands in $\mathrm{LaFeAsO}$. In addition, the Bloch functions of these bands possess the same symmetry as the related Bloch functions in $\mathrm{LaFeAsO}$. As a consequence, also in the band structure of $\mathrm{LiFeAs}$ we cannot detect a superconducting band and, hence, also LiFeAs cannot develop a stable superconducting state in the space group $P 4 / n m m$.

\section{DISTORTED LiFeAs CAN EXHIBIT SUPERCONDUCTIVITY}

There is now both a correspondence and an apparent contradiction between the experimental observations and the predictions of the NHM: on the one hand, $\mathrm{LiFeAs}$ does not develop the magnetic order observed in $\mathrm{LaFeAsO}$ as it is, in fact, not allowed within the NHM. On the other hand, undoped LiFeAs shows superconductivity, but theoretically the symmetry of the Bloch functions in the band structure of LiFeAs does not permit a superconducting state in the space group $P 4 / \mathrm{nmm}$. However, this contradiction can be healed by a (small) distortion of the crystal structure reducing the symmetry of $P 4 / \mathrm{nmm}$.

In fact, several distortions of LiFeAs would allow a stable superconducting state in the framework of the NHM. The chemical analogy of LiFeAs to LaFeAsO, however, suggests that also in LiFeAs the symmetry of the system is reduced by a (slight) displacement of the Fe atoms as it was experimentally observed in $\mathrm{LaFeAsO}$ [3]. Indeed, the displacement of the Fe atoms as depicted in Fig. 22 (a) as well as the displacement depicted in Fig. 2 (b) changes the symmetry of the Bloch functions in such a way that a stable superconducting state becomes possible. The dis- placement in Fig. 2 (a) realizes the space group $P m m 2$ (25) leaving the translation symmetry unchanged but reducing the point group $D_{4 h}$ of $P 4 / n m m$ to $C_{2 v}$. The displacement depicted in Fig. 2 (b), on the other hand, realizes the space group $P 4_{2} / n m c$ (137) leaving the point group unchanged but modifies the translation symmetry of the crystal. The superconducting band related to Fig. 2 (a) was already considered in Ref. [8]. The superconducting band related to the displacement depicted in Fig. 2 (b) is not published thus far. We shall identify it once the displacement in Fig. 2 (b) is experimentally confirmed.

\section{SUMMARY}

We propose that LiFeAs undergoes a structural distortion below a temperature $T_{s}$ higher than (or at least equal to) the superconducting transition temperature $T_{c}$. The distorted system (below $T_{s}$ ) exhibits an interaction between the electron spins and crystal-spin-1 bosons which produces below $T_{c}$ stable Cooper pairs [8]. Several distortions in LiFeAs are conceivable, the experimental observations [3] on $\mathrm{LaFeAsO}$, however, suggest that also in $\mathrm{LiFeAs}$ the distortion is realized by a displacement of the Fe atoms as depicted in Fig. 2 (a) or (b).

\section{ACKNOWLEDGMENTS}

We are indebted to Tünde Erdie from the Höchstleistungsrechenzentrum Stuttgart for kindly running the FHI-aims program and to Ernst Helmut Brandt for valuable discussion. 


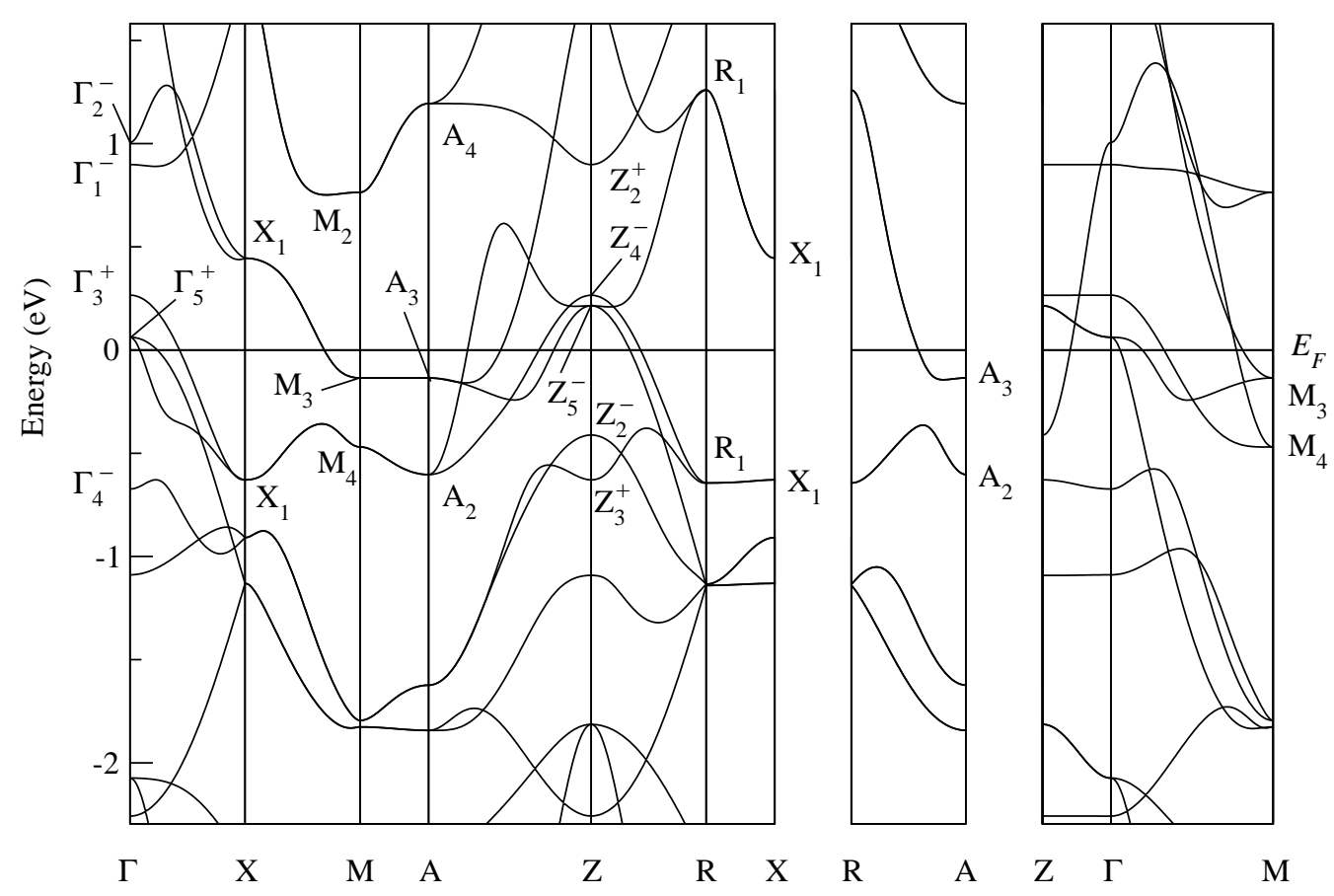

FIG. 1. Band structure of tetragonal LiFeAs as calculated by the FHI-aims program [16, 17]. The symmetry labels are determined by the authors and defined in Ref [7].

\section{$\bigcirc \mathrm{Fe}$ atoms \\ $\nabla \Delta$ directions of \\ the displacements}

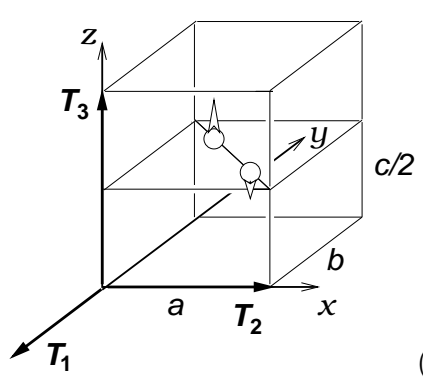

(a)

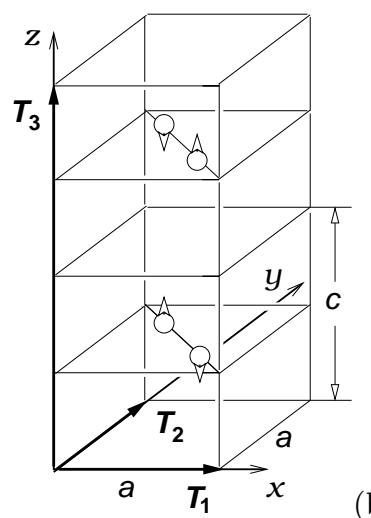

(b)

FIG. 2. Proposed unit cells and lattice vectors of two distorted LiFeAs crystals allowing a stable superconducting state. Both distortions are produced by a mere displacement of the Fe atoms. For reasons of clarity, only the Fe atoms are shown. $a, b=a$, and $c$ stand for the lengths of the unit cell of tetragonal undistorted LiFeAs (with the space group $P 4 / n m m$ ), and $\boldsymbol{T}_{1}, \boldsymbol{T}_{2}$, and $\boldsymbol{T}_{3}$ denote the basic translations of the corresponding Bravais lattice. (a) The displacement is periodic with the lattice vector $T_{3}$ of undistorted LiFeAs and realize the orthorhombic space group Pmm2 (25). The Fe atoms are displaced in $\pm z$ direction with different amounts in the unit cell. (b) The unit cell of undistorted LiFeAs is doubled in $z$ direction. The Fe atoms still are displaced in $\pm z$ direction, where a translation by the lattice vector $T_{3}$ of undistorted LiFeAs effects an inversion of the displacement. This displacement of the Fe atoms realizes the tetragonal space group $P 4_{2} / n m c$ (137) possessing the same point group $D_{4 h}$ as the space group $P 4 / n m m$ of undistorted LiFeAs. 
1. J. H. Tapp, Z. Tang, B. Lv, K. Sasmal, B. Lorenz, P. C. W. Chu, and A. M. Guloy, Phys. Rev. B 78, 060505 (2008).

2. Y. A. Izyumov and E. Z. Kurmaev, Physics-Uspekhi 51, 1261 (2008).

3. C. de la Cruz, Q. Huang, J. W. Lynn, J. Li, W. R. II, J. L. Zarestky, H. A. Mook, G. F. Chen, J. L. Luo, N. L. Wang, and P. Dai, nature 453, 899 (2008).

4. T. Nomura, S. W. Kim, Y. Kamihara, M. Hirano, P. V. Sushko, K. Kato, M. Takata, A. L. Shluger, and H. Hosono, Supercond. Sci. Technol. 21, 125028 (2008).

5. S. Kitao, Y. Kobayashi, S. Higashitaniguchi, M. Saito, Y. Kamihara, M. Hirano, T. Mitsui, H. Hosono, and M. Seto, J. Phys. Soc. Japan 77, 103706 (2008).

6. Y. Nakai, K. Ishida, Y. Kamihara, M. Hirano, and H. Hosono, J. Phys. Soc. Japan 77, 073701 (2008).

7. E. Krüger and H. P. Strunk, J. Supercond. 24, 2103 (2011), arXiv: $1104.0257 \mathrm{v} 1$.

8. E. Krüger and H. P. Strunk, J. Supercond. DOI 10.1007/s10948-012-1408-5

arXiv:1112.3169v1.
9. E. Krüger, Phys. Rev. B 40, 11090 (1989).

10. E. Krüger, Phys. Status Solidi B 85, 261 (1978).

11. Y. Kamihara, T. Watanabe, M. Hirano, and H. Hosono, J. Am. Chem. Soc. 130, 3296 (2008).

12. G. F. Chen, Z. Li, G. Li, J. Zhou, D. Wu, J. Dong, W. Z. Hu, P. Zheng, Z. J. Chen, H. Q. Yuan, J. Singleton, J. L. Luo, and N. L. Wang, Phys. Rev. Lett. 101, 057007 (2008).

13. X. H. Chen, T. Wu, G. Wu, R. H. Liu, H. Chen, and D. F. Fang, Nature 453, 761 (2008).

14. H.-H. Wen, G. Mu, L. Fang, H. Yank, and X. Zhu, Europhys. Lett. 82, 17009 (2008).

15. J. Dong, H. J. Zhang, G. Xu, Z. Li, G. Li, W. Z. Hu, D. Wu, G. F. Chen, X. Dai, J. L. Luo, Z. Fang, and N. L. Wang, Europhys. Lett. 83, 27006 (2008).

16. V. Blum, R. Gehrke, F. Hanke, P. Havu, V. Havu, X. Ren, K. Reuter, and M. Scheffler, Computer Physics Communications 180, 2175 (2009).

17. V. Havu, V. Blum, P. Havu, and M. Scheffler, Computer Physics Communications 228, 8367 (2009). 